Stéphane André

Yves Meshaka

Christian Cunat

\section{Rheological constitutive equation of solids: a link between models based on irreversible thermodynamics and on fractional order derivative equations}

Received: 10 July 2002

Revised: 27 January 2003

Accepted: 19 February 2003

Published online: 30 April 2003

(C) Springer-Verlag 2003

\begin{abstract}
In this paper we focus on the rheological problem of defining a constitutive equation for viscoelastic materials. In this simple case, we show that writing the dissipative component of the observable response to a given excitation as the result of multiple internal processes working for equilibrium recovery (flux of internal hidden variables), can yield a recursive series in time. This can be obtained when use is made of the theorem of created entropy equipartition as a model for fluctuation regression. A distribu-
\end{abstract}

tion (spectrum) for relaxation times naturally follows. The model thus obtained reflects the concept of a hierarchically constrained dynamic behavior. The conclusion is that the operator of non-integer differentiation in time applied to field variables can also be recovered from pure thermodynamic considerations.

\author{
Keywords Relaxation \\ phenomena - Irreversible \\ thermodynamics $\cdot$ Fractional \\ calculus $\cdot$ Fractal dynamics \\ Rheology $\cdot$ Viscoelasticity
}

\section{Introduction}

Damping effects in materials produce a dissipative loss of energy due to internal microscopic mechanisms that could be referred to as friction or viscous effects, thus making the distinction with purely elastic behaviors. This loss of energy has to be taken into account either towards the resulting temperature elevation that may modify the properties of the material or towards the design of structures if damping effects are sought. Research efforts were first devoted to elaborate macroscopic constitutive equations that correctly describe the dynamic behavior of such materials, relating input stress and observed deformation response (or vice versa) through material constants allowing for a classification of various materials to be made (Ferry 1980; Tschoegl 1989). Then, besides the works done by physicists for a deeper knowledge of the underlying microscopic mechanisms, approaches have been developed in two distinct directions. For the first one, the objectives were to extend the dynamic validity of the identified laws to the thermodynamic effects resulting from entropy production (thermomechanic coupling). For the second one, the objectives were to handle the multi scale nature of the physical processes involved with as few parameters as possible. The inferred laws was expected to be intrinsic, that is independent of the excitation function (or the frequency spectrum of its Fourier transform). Of course, in both approaches, the production of macroscopic relationships linking controlled and measurable variables was the aim.

The macroscopic theory of the Thermodynamics of Irreversible Processes (TIP) is the conceptual framework that offers the possibility to formulate phenomenological constitutive equations of the rheology of materials. This can involve, among others, the thermomechanic coupling discussed above. If we add the intensive temperature variable to the dynamic ones, it is possible to obtain behavior laws that would allow us, for example, to estimate physical parameters from dynamic experiments that also reflect the temperature change occurring in the sample. This can be measured elsewhere, with 
infrared based detection systems for example (Chrysochoos and Peyroux 1998; Tauchert 1967; Dillon 1962). One way for thermodynamic models to account for irreversible processes inducing memory effects consists in an increase of the thermodynamic fields through the introduction of (unknown) internal state variables acting through the thermodynamic potentials (Meixner 1949; Biot 1958; Kluitenberg 1962; Schapery 1966; Coleman and Gurtin 1967; Prigogine 1968; Nowick and Berry 1972; Allen 1985) and Kuiken 1994 for a recent application to rheology. They lead to the so-called incomplete systems widely employed to model relaxation phenomena. Anelastic (non-zero entropy production) responses are obtained through the summation of the contribution of one or more internal mechanisms or processes, each controlled by its own relaxation time.

Fractional Derivative Models (FDM) constitute a more recent direction of research especially in the field of rheology. Since the first attempts (Rouse 1953) to found these models on the basis of the description of the underlying mechanisms at microscopic level, one can also quote the stochastic-based on quantum mechanicsmodels (Bendler and Shlesinger 1985) that enable the recovery of the Kohlrausch-Williams-Watt stretched exponential model of relaxation from random walk processes (Bendler and Shlesinger 1988). More recently, there has been more and more literature in materials engineering, assessing a fractal geometrical character at microscopic level, from experimental observations (Zaiser and Hähner 1999; Balankin 1997; Kleiser and Bocek 1986; Mosolov 1994). Since dynamic phenomena occurring on a fractal space have been proved to be naturally described by fractional operators (LeMéhauté et al. 1998), one has a first theoretical basis for using them when modeling the dynamics of relaxation. However, the main reason for the use of FDM in rheology is without doubt their successfully experienced aptitude to reproduce the dynamic behavior of many modern engineering materials in a very efficient manner, both in terms of precision and of larger (several decades) frequency range, with very few parameters. Recent and growing literature exists on the subject (see, e.g., Friedrich et al. 1999; Schiessel et al. 1995; Mainardi 1994; Nonnenmacher and Glöckle 1991; Glöckle and Nonnenmacher 1991; Bagley 1989, 1991; Padovan and Guo 1988; Koeller 1984; Rogers 1983) and some (Heymans and Bauwens 1994; Schiessel and Blumen 1993) show the connections with hierarchically self-similar models relying on the classical base elements that are the spring and the dashpot. The non-integer derivative exponent for the time derivatives may be found from a defined set of elastic moduli and viscosity parameters. These models have been referred to sometimes incorrectly as "Fractal" models. They have received a growing interest mainly when very empirical old models expressed in terms of non integer power law formulations (Nutting 1921;
Gemant 1936) have been reinvested in the light of a mathematical interpretation of the integral Boltzmann formulation of constitutive laws with power law type memory kernel in terms of the Riemann-Liouville operator of diff-integration. First introduced as a mathematical trick to generalize constitutive laws more able to portray fading memory, fractional derivative models have raised the important question of establishing admissibility criteria towards their thermodynamic consistency and have led to numerous studies in that direction (Lion 1997; Bagley and Torvik 1986).

As a consequence, connections between TIP and FDM models could not be avoided and have already been pointed out (Kuiken 1994). So it is in the paper of Enelund and Olsson (1999), where the authors suggest that interpreting the whole spectrum of time constants of several processes in one field may lead the dissipative response - expressed as a sum of decaying exponentials - to be advantageously replaced by a fractional order derivative applied to a single field variable. Consequently, it is interesting to mention that, compared to Williams's synthetic paper in the early 1960s (Williams 1964) about viscoelastic behavior laws, this paper will simply add a small thermodynamically based ingredient that will achieve the convergence of the two approaches mentioned above.

In this paper, we first begin (next section) with a presentation of a TIP based model, referred to through the paper as the DNLR model (Distribution of NonLinear Relaxations) and developed at LEMTA (Cunat 1988, 1991, 2001). As its name explicitly reveals, this model goes a step further than the above-mentioned models as it makes use of the fluctuation-dissipation theorem of Prigogine (1968) to define a spectrum for the relaxation processes in the vicinity of the equilibrium and allocate them a distribution of their respective weight on the global observable response. This model has been thoroughly presented in other papers in various applications in solid mechanics such as viscoelasticity and plasticity (see, e.g., Ayadi et al. 1999; Faccio-Toussaint et al. 2001; Rahouadj and Cunat 2001a), thermostimulated creeping (Haddad 1996), aging (Aharoune et al. 2001; Rahouadj and Cunat 2001b), as well as in thermochemical applications like glass transition modeling (Cunat 1985). Therefore we will only present the necessary bases of this model to establish a link with FDM.

Following this, we will show that the distribution law for relaxation times obtained from pure thermodynamic considerations lead the DNLR approach to a modal recursive description of the dynamic of relaxation. The equipartition theorem of the created entropy accounting for spontaneous fluctuation regressions appears as the recursive law that has to be introduced in a hierarchical model where each process is given a relaxation time $\tau$. As mentioned in the abstract, this work refers to the establishment of constitutive equations for viscoelastic 
media. Microscopic arguments for modeling relaxation in such media through the introduction of a hierarchy of times have been given if stochastic processes for defect migrations (defect-diffusion models presented for example by Stastna et al. 1990) are considered.

Our purpose will then be to demonstrate that, thanks to the concept of recursivity (Oustaloup 1995; Shlesinger and Klafter 1986; Oldham and Zoski 1983), FDM can be completely driven or recovered through the DNLR approach. In other words, the order of fractional differentiation for the physical processes involved appears as the result of a modeling of the regression of fluctuations according to an equal entropy production for each of them. Illustrating results are given through the modeling of the response of viscoelastic materials subjected to a step of constant load (creep test) and to a tensile test of constant strain rate.

Finally, we will summarize the paper and discuss perspectives for future work.

\section{The D.N.L.R. thermodynamic approach}

Thermodynamical constitutive laws

The DNLR approach used to derive thermodynamically consistent behavior laws has been thoroughly detailed in previous papers (Cunat 1988, 1991, 2001) and we skip here the details to express the results rapidly. A wellsuited thermodynamic potential function $\Psi_{\mathrm{k}}$ is first selected to describe the dynamics of both instantaneous and irreversible phenomena occurring in a representative volume in local equilibrium (no gradients of any intensive variables). 'Hidden' internal variables (a field of processes $\bar{z}$ ) are also made dependent variables of this thermodynamic generalized potential. The following set of equations is then obtained:

$\underline{\dot{\beta}}=\underline{\underline{a}}^{u} \underline{\dot{\gamma}}+\underline{\bar{b}} \overline{\dot{z}}$

$\overline{\dot{A}}=-\underline{b}^{T} \dot{\gamma}-\overline{\bar{g}} \overline{\dot{z}}$

Such a formulation can be found elsewhere (Nowick and Berry 1972). It has also been used by Lesieutre and Mingori (1990) and falls into the general framework exposed by Maugin and Muschik (1994).

The lower bars refer to state variables and the upper bars, to the dissipation variables.

$a^{u}=\frac{\partial^{2} \Psi_{k}}{\partial \gamma_{\gamma} \partial \gamma_{m}}$ is the square stability matrix of Tisza (19)6), constructed from the instantaneous physical properties linking the unrelaxed observed responses $\beta$ to command excitations $\gamma$ and integrating couplings between phenomena.

$\underline{\bar{b}}=\frac{\partial^{2} \Psi_{k}}{\partial z_{j} \partial \gamma_{m}}$ is a rectangular matrix figuring the couplings between state and dissipative variables.

$\overline{\bar{g}}=\frac{\partial^{2} \Psi_{k}}{\partial z_{i} \partial z_{j}}$ is the square dissipative matrix.
One must stress that this formulation comes from the extension of the Gibbs-Duhem relation through states of internal non-equilibrium. The interesting aspect of the model is the presence of the second equation (Eq. 1b) that corresponds to the evolution equation of the non equilibrium forces $\bar{A}$ - affinities introduced by De Donder (1920) - under the effect of the state command variables $\gamma$ and of their conjugated internal variables $\bar{z}$ working for recovering a relaxed state.

This state, denoted by the superscript $r$, is obviously characterized by $\quad \overline{\dot{\mathrm{A}}}=0$ and $\overline{\mathrm{z}}=\overline{\mathrm{z}}^{\mathrm{r}}$. The dissipation variables definition respect the positivity of the production of entropy which is obtained as $T \dot{S}_{i}=\bar{A} \overline{\dot{z}}=$ $\sum_{j} A_{j} \dot{z}_{j} \geq 0$. The overall entropy produced corresponds to the summation (extensive character) of that produced by each individual process.

A hypothesis of thermodynamic linearity about the relaxed state, and of proportional relation between fluxes (velocity) of hidden variables and forces (equipresence principle of Onsager), leads to the following equations:

$\bar{A}(t)=-\overline{\bar{g}}\left(\bar{z}(t)-\bar{z}^{r}\right)$

$\overline{\dot{z}}=\overline{\bar{L}} \bar{A}$

where $\overline{\bar{L}}$ is referred to as the Onsager matrix.

Combining these two equations yields

$\overline{\dot{z}}=-\overline{\bar{L}} \overline{\bar{g}}\left(\bar{z}(t)-\bar{z}^{r}\right)=-\overline{\bar{\tau}}^{-1}\left(\bar{z}(t)-\bar{z}^{r}\right)$

where $\overline{\bar{\tau}}^{-1}$ is the operator of relaxation times. This matrix can be diagonalized according to the demonstration given by Meixner (1949), showing the existence of a modal base that allows to write all the processes in an uncoupled manner. The physical sense of the underlying elementary mechanisms of reorganization at the molecular state is lost and we then prefer talking about processes, but the approach makes the mathematical treatment easier. The possibility of this base change is justified when the same overall entropy produced is preserved.

Until now this approach has suffered from a lack of practical applicability since the internal variables are unknown. This point is overcome when the dissipative or anelastic component $\dot{\beta}^{(a)}=\bar{b} \bar{z}$ of the observable response $\dot{\beta}$ in Eq. (2a) is rewritten in terms of the difference (gap) between the present value of the response and the value taken at relaxed state. Writing Eqs. (1a) and (1b) for the current and relaxed states produces the discretized equation

$\Delta \beta_{n, j}-\Delta \beta_{n, j}^{r}=b_{n, j}\left(\Delta z_{j}-\Delta z_{j}^{r}\right)$

where $\mathrm{n}$ denotes a particular observable variable (e.g., a component of the strain tensor) and $\mathrm{j}$, the $\mathrm{j}$-th internal 
process. This procedure corresponds exactly to the formalism introduced by Onsager (1931) to develop the theory of thermodynamic fluctuations around an equilibrium state (see Kuiken 1994; De Groot and Mazur 1962 for a detailed presentation of this matter).

Then Eq. (1a) becomes

$\underline{\dot{\beta}}=\underline{\dot{\beta}}^{(e)}+\underline{\dot{\beta}}^{(a)}=\underline{\underline{a}}^{u} \underline{\dot{\gamma}}-\left(\underline{\beta}-\underline{\beta}^{r}\right) \bar{\tau}^{-1}$

where $\dot{\tau}$ is now a vector of times for the relaxation modes, in the space of dissipations. It is the component $\dot{\beta}^{(a)}$ here expressed with the scalar product $\left(\underline{\beta}-\beta^{r}\right) \bar{\tau}^{-1}$ that will be the object of a mathematical analysis to reveal the fractional derivative operator as suggested by Enelund and Olsson (1999).

\section{Distribution of non-linear relaxations}

The operator $\dot{\tau}^{-1}$ defined above has been expanded over a set of uncoupled normal modes for the relaxation mechanisms. The generality of this thermodynamic approach led Cunat (1988) to find a way to define a distribution of times for all the relaxation processes, thanks to the equipartition theorem of the created entropy (De Groot and Mazur 1962; Prigogine 1968) established in a linear range, and to associate the corresponding weight it imposes on the global behavior with each process.

This theorem states that, near the equilibrium, each dissipative mode $\mathrm{j}$ produces on average, the same dissipation

$\left\langle\frac{\partial \Delta S_{i}}{\partial \Delta z_{j}} \Delta z_{j}\right\rangle=-k_{B} \delta_{i j}<0$

where $\mathrm{k}_{\mathrm{B}}$ is the Boltzmann constant. This special case of describing the regression of fluctuations near the equilibrium state corresponds to distributions of Gaussian type for each mode, whose variances correspond to the weight $\mathrm{p}_{j}^{0}$ associated with each process of regression.

Some developments lead to define the Distribution of Non-Linear Relaxations with the following two relations:

$p_{j}^{0}=\frac{\Delta z_{j}}{\sum_{k=1}^{N} \Delta z_{k}}=\frac{\sqrt{\tau_{j}}}{\sum_{k=1}^{N} \sqrt{\tau_{k}}}=B \sqrt{\tau_{j}}$

B being defined as $B=1 / \sum_{k=1}^{N} \sqrt{\tau_{k}}$ as a result of the
normalization condition

$\sum_{j=1}^{N} p_{j}^{0}=1$

In practice, it is completely determined by three parameters:
D The number of decades of the spectrum range and arbitrarily logarithmically equally spaced

$\mathrm{N}$ The number of processes that are considered

$\tau_{\max }$ The largest relaxation time that is considered. $\tau_{\max }=\tau_{\text {macro }}$, is the largest measure for the time, in which the deviations of relaxed (equilibrium) state are macroscopically observable (Kuiken 1994)

It is important to note that this spectrum is strictly bounded between two relaxation times. It generalizes the original idea of Kovacs et al. (1979) for the description of the kinetics of volume recovery in the glassy state.

As an example we show the DNLR spectrum for $\mathrm{D}=6$, $\mathrm{N}=50$ processes and $\tau_{\max }=1000 \mathrm{~s}$ on the curve in Fig. 1 .

One-dimensional mechanical constitutive equations - Laplace transformed expression and integral formulation

Equation (5) directly yields the corresponding constitutive equations, once the command and response variables have been defined. For instance, if only the mechanical behavior is sought in isothermal conditions and if an input stress is considered, we have for a single process of relaxation $\mathrm{j}$,

$\dot{\varepsilon}_{j}=\dot{\varepsilon}_{j}^{(e)}+\dot{\varepsilon}_{j}^{(a)}=s_{j}^{u} \dot{\sigma}_{j}-\frac{\varepsilon_{j}-\varepsilon_{j}^{r}}{\tau_{j}^{\varepsilon}}$

The relaxed strain is $\varepsilon^{\mathrm{r}}{ }_{\mathrm{j}}=\mathrm{s}_{\mathrm{j}}^{\mathrm{r}} \sigma=\mathrm{p}_{0}^{\mathrm{j}} \mathrm{s}^{\mathrm{r}} \sigma$ and $\mathrm{s}^{\mathrm{u}}$ and $\mathrm{s}^{\mathrm{r}}$ denote the unrelaxed and relaxed compliance of the matter, inverse of the moduli $E^{u}$ and $E^{r}$. Equation (8) is the well known constitutive equation for the Zener's Standard Linear Solid (e.g., Tschoegl 1989, p 147; Kuiken 1994, p 306).

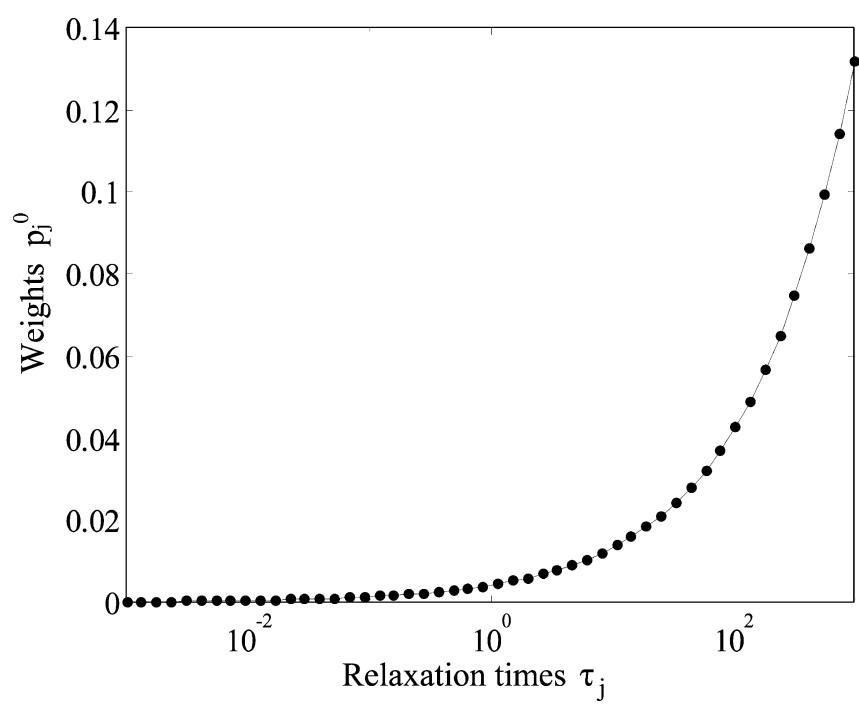

Fig. 1 Weights and relaxation times distribution of the DNLR model 
$\tau^{\varepsilon} \mathrm{j}$ is the characteristic time of the $\mathrm{j}$-th dipole resulting from the connection of a spring of compliance $s^{a}{ }_{j}=s^{r}{ }^{-}$ $\mathrm{s}_{\mathrm{j}}^{\mathrm{u}}=\mathrm{p}_{\mathrm{j}}^{0}\left(\mathrm{~s}^{\mathrm{r}}-\mathrm{s}^{\mathrm{u}}\right)=1 / \mathrm{E}^{\mathrm{a}}{ }_{\mathrm{j}}=\mathrm{p}_{\mathrm{j}}^{0} \mathrm{~s}^{\mathrm{a}}$ in parallel with a dashpot of viscosity $\eta_{\mathrm{j}}$, thus making $\tau_{\mathrm{j}}^{\varepsilon}=\tau_{\mathrm{j}}=\mathrm{s}_{\mathrm{j}}^{\mathrm{a}} \eta_{\mathrm{j}}$.

The analogous representation of the DNLR thus corresponds to a generalized Voigt model (N KelvinVoigt units connected in series) as shown in Fig. 2.

Focusing our attention on the anelastic (a) contribution of the total deformation rate, we can write

$\dot{\varepsilon}_{j}^{(a)}=\frac{\varepsilon_{j}^{r}-\varepsilon_{j}}{\tau_{j}^{\varepsilon}}=\frac{s_{j}^{r} \sigma-s_{j}^{u} \sigma-\varepsilon_{j}^{(a)}}{\tau_{j}^{\varepsilon}}$

In Laplace space, Eq. (9) leads to the following admittance (susceptibility) of the system describing only the anelastic part of the response:

$$
\begin{aligned}
\frac{\overline{\dot{\varepsilon}}^{(a)}(s)}{\bar{\sigma}(s)} & =Y_{a n}(s)=\frac{\sum_{j} \overline{\dot{\varepsilon}}_{j}^{(a)}(s)}{\bar{\sigma}(s)} \\
& =\sum_{j} \frac{s_{j}^{a} s}{1+\tau_{j} s}=s^{a} \sum_{j} \frac{p_{j}^{0} s}{1+\tau_{j} s}
\end{aligned}
$$

This admittance corresponds exactly to the one that can be obtained in the field of electronic circuitry when considering a scheme of dipoles connected in parallel, each being made from a resistance and a capacitor wired in series. The intensity or current (I) corresponds to the rate of deformation $(\dot{\varepsilon})$, the voltage, to the mechanical stress, the dashpot of viscosity $\eta$ being the element that carries the irreversibility and the heat dissipation $(\mathrm{R}$ in the case of a Joule effect) thus making the dissipated power $\mathrm{P}=\eta \dot{\varepsilon}^{2} \equiv \mathrm{RI}^{2}$.

The D.N.L.R formalism can be related to the convolution integral formulation of the Boltzmann type as in general all thermodynamic models based on a description with internal variables (Maugin and Muschik 1994). For the above example, the fading memory kernel has to be represented by a series of exponentially decaying kernels:

$$
\begin{aligned}
\varepsilon^{(a)}(t) & =\int_{0}^{t} \sigma(t-\tau) \frac{d J}{d \tau} d \tau \\
& =\int_{0}^{t} \sigma(t-\tau) \sum_{j=0}^{N-1} \frac{s_{j}^{a}}{\tau_{j}} \exp \left(-\tau / s_{j}^{a} \eta_{j}\right) d \tau
\end{aligned}
$$

where $\mathbf{J}(\mathrm{t})$ is the retardation function and $\mathrm{s}_{\mathrm{j}}^{\mathrm{a}} \eta_{\mathrm{j}}=\tau_{\mathrm{j}}$ corresponds to the time constant associated with the $\mathrm{j}$-th element of the cascade representation.

It is important to recall here that the Fractional Derivative Models that will be discussed later have been essentially derived from the integral formulation, the memory kernel being related to a series of power functions of time or directly to non-integer power law decaying functions. Here, FDM will be recovered with the formulation of the memory kernel in terms of a discrete summation.

\section{Recursivity of the DNLR approach}

The specific characteristic of the model presented above (Fig. 2) lies in the geometric progression that rules the evolution of both parameters $\mathrm{E}_{\mathrm{j}}^{\mathrm{a}}$ or $\mathrm{s}_{\mathrm{j}}^{\mathrm{a}}$ and $\eta_{\mathrm{j}}$ as a result of using the theorem of equipartition of the created entropy rate. The DNLR model then belongs to the second class of relaxation theories as exposed by Shlesinger and Klafter (1986) and named "hierarchically constrained dynamics".

This will be the object of the first section to define clearly what will be called the recursive parameters of the model. Two different sets of parameters $(\alpha, \beta)$ and $(p, q)$ can be defined depending on whether one decides to describe the analogic representation respectively in terms of the (modulus/viscosity) pair or with the more general (or universal) set which is the (relaxation time/ 'distance' from relaxed state) pair. The latter will then be the one used for further developments.

In the second section, we will start from the expression of the admittance function established in the previous section to give its corresponding expression in the temporal domain, in terms of the new recursive parameters. This will illustrate the concept of recursive series in time as defined by Oustaloup (1995).

Later we will calculate the recursive factors in the case of the DNLR model and give the characterization of the anelastic (dissipative) admittance in the frequency domain using a Bode diagram representation (Oustaloup 1995; Rogers 1983).

Finally, we will transform the summations of the recursive series into a ratio of factored polynomials, and consequently we'll recover a characterization in terms of zeros and poles (relaxation times).
Fig. 2 Generalized Voight model

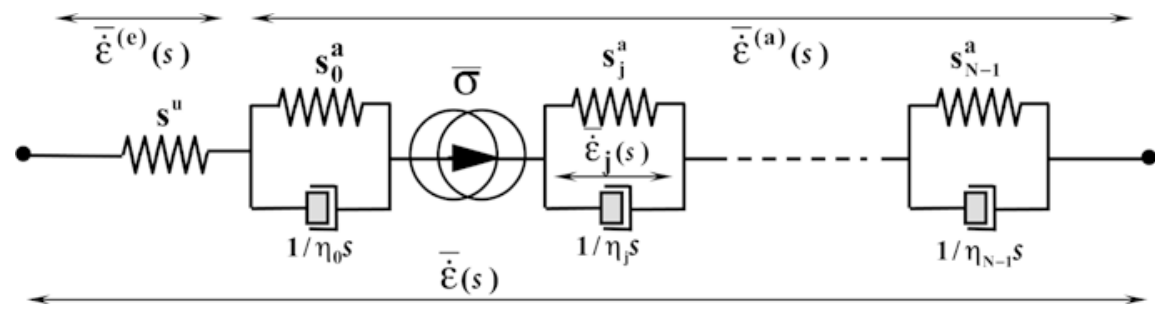


Recursivity of the parameters of the DNLR model

In the following, we consider the series in Laplace space of the normalized admittance (Eq. 10):

$Y_{a n}^{*}(s)=\frac{Y_{a n}(s)}{s^{a}}=\sum_{j} \frac{p_{j}^{0} s}{1+\tau_{j} s}$

From Eqs. (7a) and (7b) it is easy to recover the following ratios, that we assume to be constant. They will be proved so later in the text:

$\frac{s_{j+1}^{a}}{s_{j}^{a}}=\frac{1}{\beta}=\frac{p_{j+1}^{0}}{p_{j}^{0}}=\frac{p}{q^{2}}$
$\frac{\eta_{j+1}}{\eta_{j}}=\frac{1}{\alpha}=\frac{q}{p}$

Recursive factors

$\alpha$ and $\beta$ are the recursive factors associated to a description in terms of the above set of physical properties (E or $\mathrm{s}, \eta$ ) of the material. If one arbitrarily considers to choose both $\alpha$ and $\beta>1$ (which implies both $p$ and $\mathrm{q}>1$ ), then increasing rank $\mathrm{j}$ means that we will have decreasing values of the viscosity $\eta$ starting from a maximum value $\eta_{0}$ and also decreasing values of the compliance $\mathrm{s}^{\mathrm{a}}$ from the maximum value $\mathrm{s}_{0}{ }_{0}$.

The introduction of the additional set of recursive factors $\mathrm{p}$ and $\mathrm{q}$ can now be made clear by introducing the characteristic time (or characteristic frequency) associated with each rank $\mathrm{j}$ :

$\tau_{j}=s_{j} \eta_{j} \quad$ or $\quad \omega_{j}=\tau_{j}^{-1}=\left(s_{j} \eta_{j}\right)^{-1}$

Using the spectrum law defined in Eqs. (7a) and (14) along with the recursive factor definitions of Eqs. (13a) and (13b) yields

$\left(\frac{p_{j+1}^{0}}{p_{j}^{0}}\right)^{2}=\frac{\tau_{j+1}}{\tau_{j}}=\frac{1}{\alpha \beta}=\frac{1}{q}$

$\mathrm{q}$ appears then as the recursive factor for the geometric progression of relaxation times. Increasing rank $j$ induces decreasing relaxation times or increasing 'transitional' frequencies as $\omega_{\mathrm{j}+1} / \omega_{\mathrm{j}}=\mathrm{q}$. The relaxation times can be constructed from the minimum frequency $\omega_{0}=1 / \tau_{\max }$ with $\omega_{\mathrm{j}}=\mathrm{q}^{\mathrm{j}} \omega_{0}$ where $\mathrm{q}^{\mathrm{j}}$ strictly corresponds to $\mathrm{q}$ raised to the power $\mathrm{j}$.

At this point one can also retain the relationship between the two sets of recursive factors $\mathrm{q}=\alpha \beta$ thus leading to $p=\alpha^{2} \beta$. From Eqs. (13a) and (15) one finds the additional relation

$\alpha=\beta$ and $p=q^{3 / 2}$
An additional relation necessary for further calculations can be derived from the above relations that link the DNLR weights $p_{j}^{0}$ and relaxation frequencies $\omega_{j}$ in terms of the recursive $(p, q)$ set. One can show that

$\frac{\omega_{j+1} p_{j+1}^{0}}{\omega_{j} p_{j}^{0}}=\frac{p}{q} \quad$ or $\quad \omega_{j} p_{j}^{0}=\left(\frac{p}{q}\right)^{j} K$

where $\mathrm{K}=\omega_{0} \mathrm{p}_{0}^{0}$ is a constant, completely defined by the DNLR spectrum.

Values of the recursive factors for the DNLR model

For the DNLR model of the relaxation times, one determines the number of decades $\mathrm{D}$, the total number of processes $\mathrm{N}$ and the maximum relaxation time $\tau_{\max }$ that sets the first process weight $($ rank $\mathrm{j}=0)$ according to $p_{j=0}^{0}=B \sqrt{\tau_{\max }}=B / \sqrt{\omega_{0}}$.

Selecting arbitrarily equally spaced relaxation times on a $\log$ scale determines the value of the recursive factor q:

$\frac{\tau_{j+1}}{\tau_{j}}=10^{-d /(N-1)}=\frac{1}{\alpha \beta}=\frac{1}{q}$

For the spectra considered earlier as an example $(\mathrm{D}=6, \mathrm{~N}=50)$, this gives

$\mathrm{q}=1.3257$

Then, from Eqs. (15) and (16) it follows that

$\alpha=\beta=\sqrt{q}=1.1514$ and $p=q^{3 / 2}=1.5264$

Recursive series of the DNLR Admittance in time and frequency domain

In this section, we will study the dimensionless admittance function of the DNLR model (Eq. 12) representing the anelastic component as a function of the $(p, q)$ recursive factor set. One obtains

$$
\begin{aligned}
Y_{a n}^{*}(s) & =\sum_{j} \frac{p_{j}^{0} s}{1+\tau_{j} s}=K \sum_{j}\left(\frac{p}{q}\right)^{j} \frac{s / \omega_{j}}{1+s / \omega_{j}} \\
& =K \sum_{j}\left(\frac{p}{q}\right)^{j} S\left(s / \omega_{j}\right)
\end{aligned}
$$

Equation (20), when transformed back into the temporal domain and making use of the translation property relation $L\left(a f(a t)=\mathrm{F}(s / a)\right.$ applied for $\mathrm{a}=\mathrm{q}^{\mathrm{j}}$ and considering the impulse excitation $\bar{\sigma}(s)=1$, corresponds to the impulse response (subscript $\delta$ ) of the system. It can be written as follows: 
$\dot{\varepsilon}_{a n_{\delta}}^{*}(t)=K \sum_{j} p^{j} R\left(q^{j} t\right)$

In this case $R(t)$ which is called the pattern function, corresponds to the original of function $\mathrm{S}$ in Eq. (20) and is given by

$R(t)=\delta(t)-\omega_{0} e^{-\omega_{0} t}$

According to the developments exposed by Oustaloup (1995), Eqs. (21) and (22) define a so-called recursive series which exhibits a striking behavior when studied in the frequency domain (replacing $s$, Laplace variable by the pure imaginary number $\mathrm{j} \omega$ ).

Independently of the pattern function $\mathrm{R}(\mathrm{t})$, a Bode diagram reveals:

- A linear evolution of the amplitude (modulus) with slope $20 \mathrm{n} \mathrm{dB} /$ decade $(6 \mathrm{n} \mathrm{dB/oct)}$

- A constant phase (independent of frequency) which characterizes a phase blocking at the value $n \pi / 2$

The absolute value of $\mathrm{n}$ is non-integer, between 0 and 1 , and depends only on the value of the recursive factors. According to the developments exposed by Oustaloup, the non-integer value of $n$ which characterizes recursive transfer functions in Laplace or Fourier domain is obtained by

$n=\frac{1}{1+\frac{\log \beta}{\log \alpha}}=\frac{\log p}{\log q}-1$

In the case of the DNLR model the non-integer number $\mathrm{n}$ is found equal to $1 / 2$.

Equation (24) below gives the expression of the Fourier transform of the DNLR recursive series (Eq. 21), obtained from Eq. (12):

$Y_{a n}^{*}(i \omega)=\sum_{j} \frac{p_{j}^{0} i \omega}{1+i \omega \tau_{j}}=K \sum_{j}\left(\frac{p}{q}\right)^{j} \frac{i \omega / \omega_{j}}{1+i \omega / \omega_{j}}$

The Bode diagram representation of Eq. (24) in Fig. 3 gives the graphical visualization of the role played by the non-integer number $\mathrm{n}$ as the parameter of the straight lines that smoothes the evolution of the modulus and shows the phase blocking of the argument.

Two curves have been drawn on both figures, one obtained considering $\mathrm{N}=50$ processes and the other for only $\mathrm{N}=6$ processes to exhibit the 'staircase' evolution of the modulus, with ramps of slope $20 \mathrm{~dB} / \mathrm{dec}$ that alternate with horizontal segments, thus leading to a smoothing line of slope $20 \mathrm{n}=10 \mathrm{~dB} / \mathrm{dec}$ over the range of relaxation times.

This feature is a specific trend related to the frequency characterization of generalized non integer derivative operators. Indeed, by virtue of the property of Fourier transforms towards differentiation, we have
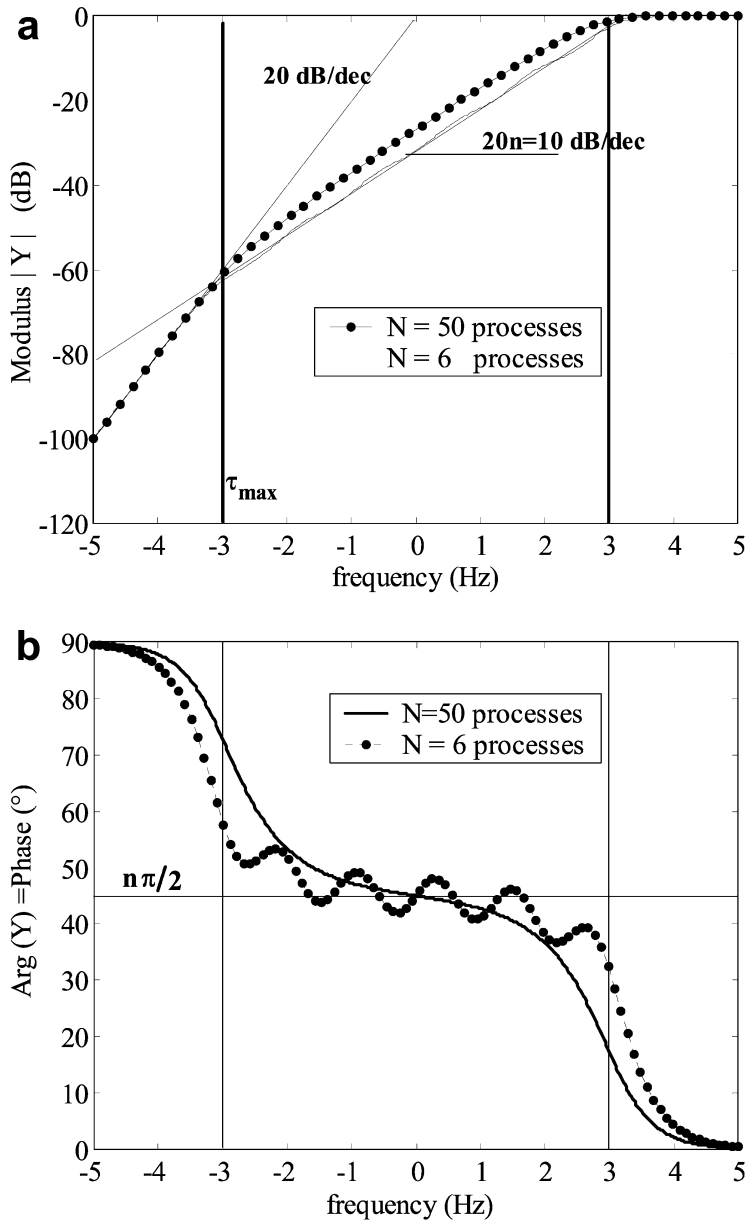

Fig. 3 a Bode diagram of $\mathrm{Y}^{*}{ }_{\text {an }}(\mathrm{i} \omega)$ : amplitude vs frequency. b Bode diagram of $\mathrm{Y}^{*}$ an $(\mathrm{i} \omega)$ : phase vs frequency

$F\left(d^{n} f(t) / d t^{n}\right)=(j \omega)^{n} \mathrm{~F}(j \omega)$. The non-integer number $\mathrm{n}$ that appeared in the frequency characterization is directly connected to the non-integer exponent of diff-integration operators representing the dynamics of the system in terms of a field variable. More details of them will be given later.

Finally, one would like to point out that the recursive definition of the impulse 'retardation' function (Eq. 21) can be directly related to the integral formulation (Eq. 11) either by use of the convolution (Faltung) theorem or in the time domain when using the property of the delta (Dirac) function.

From recursive factors to recursive sets of zeros and poles

One can apply a reduction to a same denominator of the discrete summation of the admittance given by Eq. (12). 
This leads to the following type of expression, involving a ratio of factored polynomials:

$$
Y_{a n}^{*}(s) \cong Y_{N}(s)=\frac{s}{P^{0}} \frac{\prod_{j=1}^{N-1}\left(1+\frac{s}{\omega_{j}^{\prime}}\right)}{\prod_{j=1}^{N}\left(1+\frac{s}{\omega_{j}}\right)}
$$

for which one could demonstrate that, in our case, $1 / P^{0}=\sum_{j} p_{j}^{0}=1$.

Equation (25) has been studied by Biot (1958) in order to represent rheological transfer functions for viscoelastic materials. The parameters of the model are the poles and the zeros of the fraction above. The important number of poles and zeros usually required for a satisfying fitting of experimental data and the absence of underlying physical connections for the identified parameters reduce the interest of such discretized approach. Of course a recursivity introduced here to govern the behavior of poles and zeros would overcome this difficulty, just as was the case before with an admittance in summation form. However, the operation carried out to go from Eq. (12) to Eq. (25) does not preserve the recursivity relation between the parameters: the recursivity of the poles is unchanged as the poles are the frequencies corresponding to the inverse of the relaxation times and the denominator of Eq. (25) directly corresponds to the product of the denominators of the ratios of Eq. (12). However, the recursivity in the parameters of weights of Eq. (12) is partially lost for the zeros $\omega_{j}^{\prime}$ in the operation carried out to go from Eq. (12) to Eq. (25). For this reason, the symbol 'nearly equal' $(\cong)$ has been used in Eq. (25) to indicate that it is only an approximation of Eq. (12) if ones assumes that the zeros $\omega^{\prime} \mathrm{j}$ obey the same recursion relation as the weights in the summation form of Eq. (12). One can easily verify that such a relation only holds in the central part of the frequency domain but the ratio between two consecutive zeros at each limit of the frequency domain departs slightly from that obtained in the central part. More exactly, it is only preserved in the limit of infinite relaxation times $(\mathrm{N} \rightarrow \infty)$. In this case indeed, the recursive parameter $\mathrm{q}=\alpha \beta$ tends to 1 and one could verify that consecutive zeros remain in a more constant ratio $R_{0}=\left(\omega_{j+1}^{\prime} / \omega_{j}^{\prime}\right) / q$ (Fig. 4). One then obtains

$\frac{\omega_{j}^{\prime}}{\omega_{j}}=\alpha^{\prime} \quad$ and $\quad \frac{\omega_{j+1}}{\omega_{j}^{\prime}}=\beta^{\prime}$

with $\alpha \beta=\alpha^{\prime} \beta^{\prime}$. In our special case where $\alpha=\beta$, one has also $\alpha^{\prime}=\beta^{\prime}$.

In the case of a non-infinite number of processes, Eq. (25), although it exactly conforms to Eq. (12), is only an approximation of it when the recursivity of the parameters remains unchanged. This is illustrated in

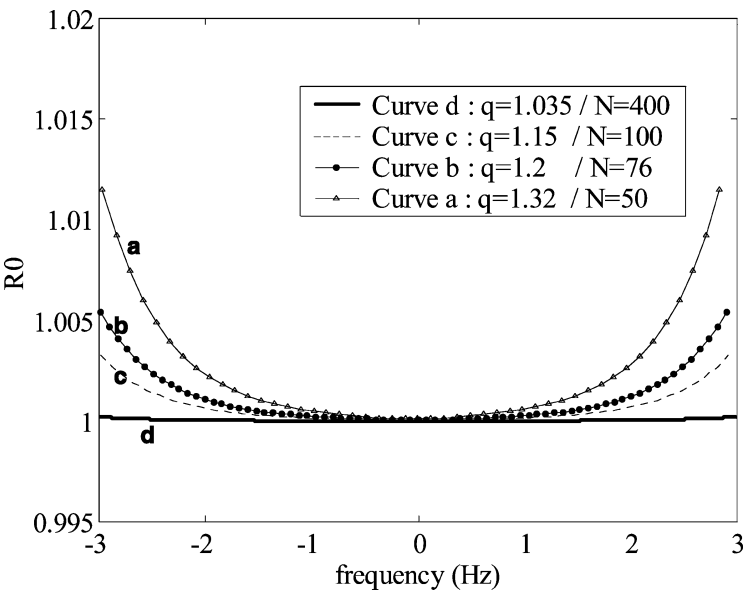

Fig. 4 Evolution of the ratio of two consecutive zeros normalized by the recursive factor $\mathrm{q}$ when considering the admittance of Eq. (45)

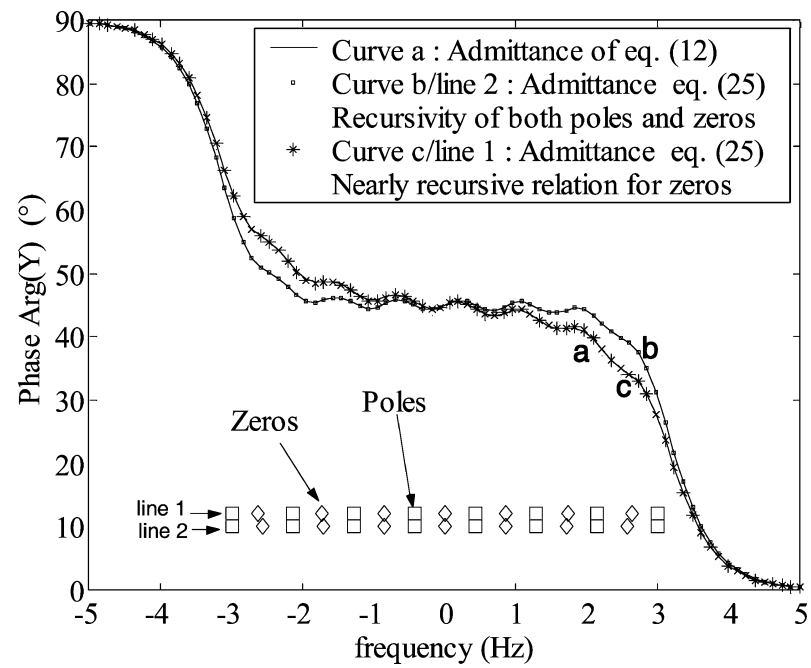

Fig. 5 Comparison of the DNLR admittance expressed as a recursive series with the admittance expressed in terms of a ratio of factored polynomials with a recursion relation between zeros and poles: phase vs frequency

Fig. 5. The same test case is considered as before with a spectrum of only eight relaxation times. One only plots here the phase curves (argument of the complex admittance given by Eq. 25) as it reveals what happens more than modulus curves.

Curve a of Fig. 5 corresponds to the admittance $\mathrm{Y}^{*}{ }_{\text {an }}$ expressed by the recursive summation of Eq. (12). Curve $\mathrm{b}$ corresponds to the admittance $\mathrm{Y}_{\mathrm{N}}$ expressed by a single ratio (Eq. 25) when preserving the recursivity on the zeros (distribution of zeros and poles shown on line 2). It is clear that this curve does not match perfectly with curve a, especially for both extremities of the frequency spectrum. 
Curve c corresponds to an admittance $\mathrm{Y}_{\mathrm{N}}$ of exactly the same form as Eq. (25) but without preserving the recursivity of zeros: the ratios between two consecutive zeros are accommodated by optimization at the extremities of the frequency range to match the original admittance. Three zeros have been identified. Due to the symmetry of the function, only the central zero remains unchanged (model with 8 poles and 7 zeros). Figure 5 shows how the distribution of zeros in line 1 (pentagram dots) is adjusted, compared to the perfectly homogeneous (recursive) distribution yield by the DNLR model shown on line 2-with equirepartition of zeros and poles - in order to make admittance of Eq. (25) matches that of Eq. (12).

In this case where $\mathrm{q}=\alpha \beta \approx 7.2$ (very far from 1 ), one finds that the first three ratios of consecutive poles (normalized by q) are $1.17,1.054,1.0146$. For the $\mathrm{N}=50$ processes generally considered for calculations in real cases, one had found before $\mathrm{q}=\alpha \beta \approx 1.32$. This value is still large compared to 1 and the representation of $\mathrm{Y}^{*}$ an with the expression of $Y_{N}$ will still be a rough approximation. This is illustrated in Fig 4 where we have considered successively $\mathrm{N}=50, \mathrm{~N}=76, \mathrm{~N}=100$, and $\mathrm{N}=400$ processes for $\mathrm{Y}^{*}{ }_{\text {an }}$. For each case, a distribution of zeros has been found (from the initial recursive distribution) that enables the admittance $Y_{N}$ to be perfectly equal to $\mathrm{Y}^{*}$ an . Figure 4 shows the ratio of two consecutive zeros, normalized by the recursive factor q. Preserving a recursive distribution for the zeros would then lead to a horizontal line at $\mathrm{R}_{0}=1$. Changing the value of the recursive factor from $\mathrm{q}=1.32(\mathrm{~N}=50)$ to $\mathrm{q}=1.035(\mathrm{~N}=400)$, curves $\mathrm{a}-\mathrm{d}$, show how large the number of processes must be for $Y_{N}$, with a recursive distribution of zeros, to be a good approximation of $\mathrm{Y}^{*}{ }_{\mathrm{an}}$. In other words, the operation that converts a summation of ratios into a single ratio of factored first order polynomials when reducing to the same denominator, is very penalizing in terms of precision when one preserves a discrete (non-rigorously continuous) recursive distribution of the zeros.

\section{From recursivity of the DNLR approach to fractional time models}

Two ways are considered in the present chapter to go from recursivity to fractional time models, according to the respective works of Oldham and Zoski (1983) and Oustaloup (1995). A dynamic evolution of a system undergoing relaxation is shown to be possibly described by two different types of diff-integration operators in time applied to the exciting continuous field variable. The first operator obtained is the 'classical' one, widely used in many works and in various combinations to construct constitutive laws describing viscoelastic behavior with accuracy. It is called the explicit non-integer differentiation and is shown to be a rough outcome for recursive dynamics that are unbounded in the spectral domain. It is directly connected to a transfer function of Cole-Cole type. The second operator is the implicit non-integer differentiation and enables us to work in a context of recursive dynamics in bounded spectral domain. Its structure allows the recovery of a Davidson-Cole relaxation transfer function. However, the common basis of the two revealed fractional operators is a clear sign of recursivity.

\section{Model of Oldham and Zoski}

As regards the first way, one starts from the works of Oldham and Zoski, about the practical realization of analog diff-integration operators by use of electronic circuits of RC types (Domino ladder scheme). They show that domino ladder networks (Parallel ResistanceCapacity cells connected in series) with geometrical progression of the values of both resistances and capacities (previously called a recursive scheme in this paper) lead the voltage output of the system to be a fractional derivative of non-integer order (between 0 and 1) of the time dependent input current. This meets the works of Heymans and Bauwens (1994) in the field of solid rheology that show how special tree-ladder or hierarchical arrangements of the classical (integer) elements (spring and dashpot) set a certain non-integer value for a representation in terms of fractional time derivatives.

Let us consider then the scheme of Fig. 2. This scheme is identical to the one considered by Oldham and Zoski provided that the springs are replaced by electrical resistors and the dash-pots by capacitors. The authors show that assuming a recursive scheme leads to the following expression for the overall deformation (voltage drop):

$\varepsilon(t)=\frac{s_{0}^{a v}}{\eta_{0}^{1-v}} \int_{0}^{t} \frac{\sigma(t-\tau)}{\tau^{v}} \sum_{j=0}^{N-1}\left(\frac{\tau \alpha^{j} \beta^{j}}{s_{0}^{a} \eta_{0}}\right) \exp \left\{-\frac{\tau \alpha^{j} \beta^{j}}{s_{0}^{a} \eta_{0}}\right\} d \tau$

where $\alpha$ and $\beta$ are the recursive factors defined in Eqs. (13a) and (13b), and parameter $v$ is defined as

$v=\frac{\ln \alpha}{\ln (\alpha \beta)}$. Of course, as the analogic schemes are identical, Eq. (27) can easily be shown to be the same as Eq. (11) using the fact that $\mathrm{q}=\alpha \beta$.

In the idealized limit of an infinite number of processes, where both $\alpha$ and $\beta$ approach unity, Oldham and Zoski show that Eq. (27) can be written thus:

$$
\begin{aligned}
\varepsilon(t) & =\frac{\pi \csc (\pi v) s_{0}^{a v}}{\ln (\alpha \beta) \eta_{0}^{1-v}} \frac{1}{\Gamma(1-v)} \int_{0}^{t} \frac{\sigma(t-\tau)}{\tau^{v}} d \tau \\
& =\frac{\pi \csc (\pi v) s_{0}^{a v}}{\ln (\alpha \beta) \eta_{0}^{1-v}} \frac{d^{v-1} \sigma(t)}{d t^{v-1}}=\varepsilon_{i d}(t)
\end{aligned}
$$


using the Riemann-Liouville definition of the fractional integral.

When Laplace transformed, one obtains

$\frac{\overline{\dot{\varepsilon}}_{i d}(s)}{\bar{\sigma}(s)}=Y_{i d}^{a n}(s)=\frac{\pi \csc (\pi v) s_{0}^{a v}}{\ln (\alpha \beta) \eta_{0}^{1-v}} s^{v}=\frac{\left(\tau_{\max } s\right)^{v}}{\eta_{0}}=\left(\tau^{\prime} s\right)^{v}$

for the ideal admittance of the system, which then leads to the simple generalized operator of differentiation d. ${ }^{v} /$ $\mathrm{dt}^{v}$ in the temporal domain. In the frequency domain, its characterization is

$Y_{i d}^{a n}(j \omega)=\left(j \tau^{\prime} \omega\right)^{v}$

The modulus and the phase are given by the following relations:

$\left|\bar{Y}_{i d}^{a n}(j \omega)\right|=\left(\tau^{\prime} \omega\right)^{v}=\left(\frac{\omega}{\omega^{\prime}}\right)^{v}$

$\operatorname{Arg}\left(\bar{Y}_{i d}^{a n}(j \omega)\right)=v \frac{\pi}{2}$

Thus the representation of the Bode diagrams lead to a modulus curve which is a straight line of slope $20 v \mathrm{~dB} /$ dec and to a phase curve which is a horizontal line of ordinate $(v \pi) / 2$ (Fig. 6a,b).

With the values of $\alpha$ and $\beta$ yielded by the DNLR model, one unsurprisingly obtains the value $v=0.5$ as non-integer exponent. Concerning the transitional frequency $\omega^{\prime}$, one obtains a value of $\tau^{\prime}=\tau_{\max } / \eta^{-v}{ }_{0}=2.1 \mathrm{e}^{-5} \mathrm{~s}$ for the test case considered in the paper.

The idealized character of this result is evident on both curves which do not match the Bode diagram of the DNLR recursive scheme due to the absence of two bounding frequencies (or times) of the relaxation spectrum.

The result obtained correspondingly for a creep experiment is shown in Fig. 7. The calculations are made in Laplace domain and the return to the temporal domain is made through numerical inversion.

The values of the parameters $\tau^{\prime}$ and $v$ of Eq. (29), calculated from the DNLR relaxation spectrum, do not lead to a good reproduction of the creep experiment. Nevertheless, when identified from the simulation yielded by the DNLR model, the simple structure of Eq. (30) is proved to be able to match the data, the identified values of $\tau^{\prime}$ and $v$ being $\tau^{\prime}=3.25 \mathrm{e}^{-5} \mathrm{~s}$ and $v=0.538$. The parsimony in the number of parameters and yet the efficiency of the fractional element characterized by the simple constitutive law $\eta \dot{\varepsilon}(\mathrm{t})=\tau^{v}\left(\mathrm{~d}^{v} \sigma(\mathrm{t}) /\right.$ $\mathrm{dt}^{v}$ ) - referred to as the spring-pot by Scott-Blair and Caffyn (1949) - to describe a great variety of responses, explains its growing success in the community.

As regards characterization in the time domain, one must first note that the constitutive law obtained $\varepsilon(\mathrm{t})=\mathrm{s}^{\mathrm{u}} \sigma(\mathrm{t})+\tau^{\prime \nu} \mathrm{d}^{\nu-1} \sigma(\mathrm{t}) / \mathrm{dt}^{\nu-1}$ meets that proposed in literature on FDM as a first generalization of basic
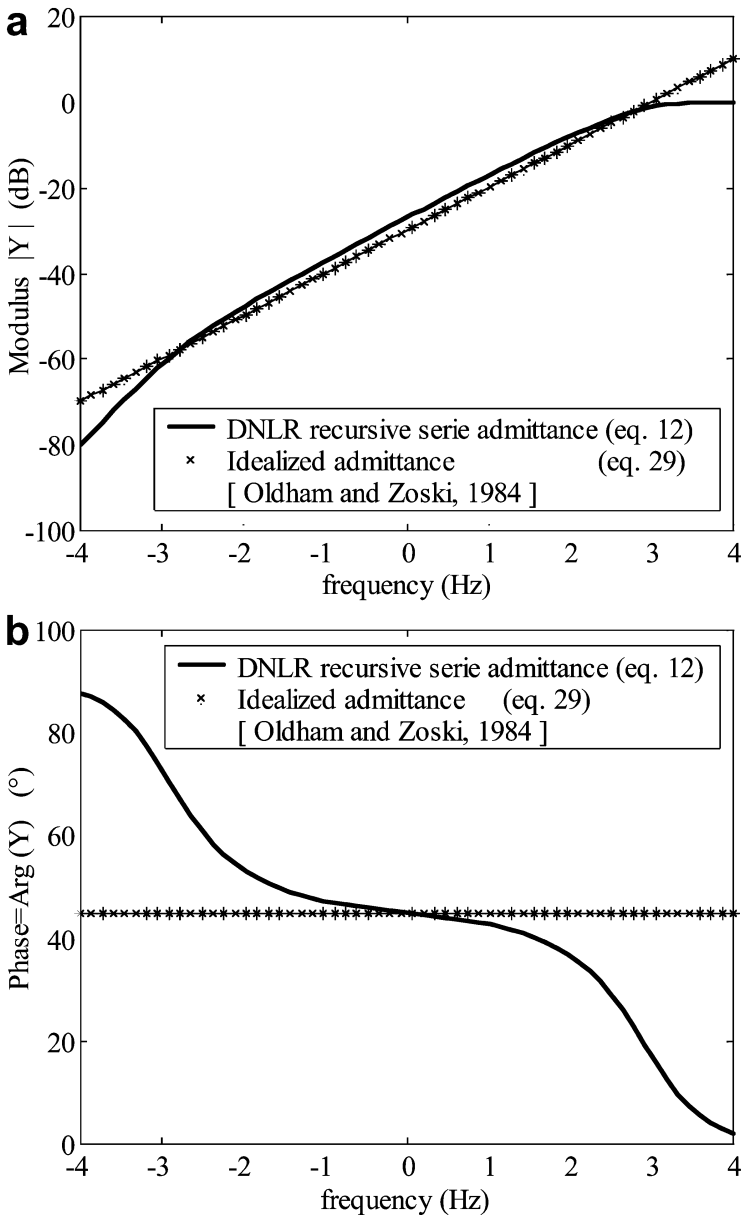

Fig. 6 a Comparison of the DNLR admittance with idealized admittance: magnitude vs frequency. b Comparison of the DNLR admittance with idealized admittance: phase vs frequency

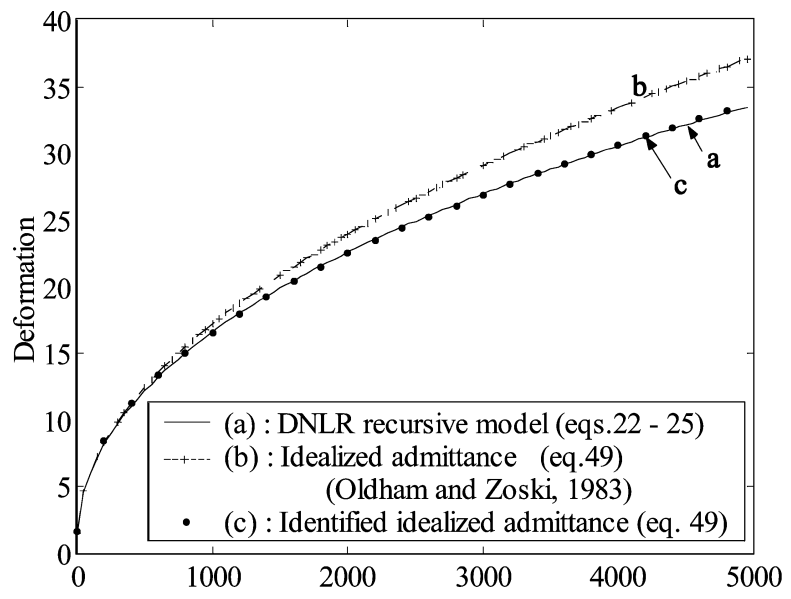

Fig. 7 Creep experiment: comparison of DNLR model with fractional model based on the idealized admittance 
analog models (substituting the single fractional element FE for the dashpot in our case; see Friedrich et al. 1999). It is mainly the necessity of introducing additional time constants in order to circumscribe a spectral range for the dynamics that, from our point of view, justifies the use of additional terms in a constitutive law. One question for example that one could address is: Why use more than one Fractional Element? Some precocious proposals of generalized fractional constitutive equations have indeed no physical backgrounds and they were sometimes discarded as soon as thermodynamic constraints were imposed (see, for example, Bagley and Torvik 1986).

One can finally observe that the inverse Laplace transform of the Laplace variable s raised to the noninteger power $v$ corresponds to the $v$-th derivative of the Dirac distribution

$L^{-1}\left(s^{v}\right)=\delta^{v}(t)=\frac{t^{-v-1}}{\Gamma(-v)} U(t)$

The integral formulation of the constitutive law is then

$\varepsilon(t)=s^{u} \sigma(t)+\tau^{\prime v-1} \int_{0}^{t} \sigma(t-u) \frac{u^{-v}}{\Gamma(1-v)} U(u) d u$

This constitutes the basic link that can be made from the classical integral formulation involving hereditary kernels whose mathematical expression can yield the fractional diff-integration operator. Schiessel et al. (1995) expressed this in a short-cut manner as "Fractional calculus: a mimicry of memory".

The idealized character of Eq. (28) did not escape the attention of Oldham and Zoski for the simple reason that they would then have needed to build a circuit with an infinite number of components. In their paper they introduced two additional $(j=-1$ and $j=N$ ) extra cells made of resistors and capacitors (springs and dashpots) in parallel, with different time constants, to define two limits for the time spectrum. The initial regular geometric sequence is then broken (at the extremities of the spectrum) and Oldham and Zoski showed that the performance of the diff-integrator thus modified could be seriously affected depending on the values of the extra components. We meet the same problem to represent the DNLR behavior by the simple non-integer derivative operator. The relaxation spectrum of the DNLR model corresponds to a regular geometric sequence (theorem of entropy equipartition) between two limiting times. One can not expect then to recover simulations yielded by the DNLR approach perfectly with a simple generalized derivative operator like the one introduced in Eq. (28) whose parameters (especially the non-integer power exponent) would be determined according to the data required to construct the DNLR spectrum. Thus, looking for the intrinsic character of a representation such as the one given by Eq. (28) for the anelastic part is simply useless even though Fig. 7 shows that the fractional operator possesses a model structure able to recover or portray 'reality'.

\section{Model of Oustaloup.}

The second way to go from recursivity to fractional time models is obtained following the work done by Oustaloup (1995) in the field of automotive damping, which shows that, in the limit of continuous spectrum, one can advantageously substitute the admittance of Eq. (25) for the more convenient model structure given by Eq. (32), where $\mathrm{n}^{\prime}$ figures as a non-integer-exponent supposed to tackle the multi-scale effect of dynamics, and $\omega_{\mathrm{b}}$ and $\omega_{\mathrm{h}}$ represent the lowest and highest frequency bounds of the relaxation spectrum:

$Y_{a n}^{*}(s)=Y_{n^{\prime}}(s)=s \frac{\left(1+\frac{s}{\omega_{h}}\right)^{n^{\prime}-1}}{\left(1+\frac{s}{\omega_{b}}\right)^{n^{\prime}}}$

This new mathematical structure for the admittance model corresponds to a smoothing of admittance $\mathrm{Y}_{\mathrm{N}}$.

In Fig. 8a,b it is shown nevertheless that this structure, although much better than that of the preceding model, Eq. (29), is not able to reproduce the characteristic of the DNLR recursive admittance perfectly. This is clearer Fig. 8b which represents the evolution of the phase of the complex admittance vs frequency. An identification of the parameters of the model has been performed on the synthetic simulations yielded by the DNLR model (phase of the admittance of Eq. 12) but without obtaining any satisfactory matching. The reconstructed admittance with the identified values of the parameters corresponds to the curves in star dots. One finds, in that case, a non-integer exponent $\mathrm{n}^{\prime}=0.5$, the exact value calculated in Eq. (23). The low and high cutoff frequencies are respectively $\omega_{\mathrm{b}}=1.7 \mathrm{mHz}$ and $\omega_{\mathrm{h}}=572 \mathrm{~Hz}$.

If one tries now to reproduce what happens in both a creep experiment and a tensile test with a ramp deformation as input signal, the results shown in Figs. 9 and 10 confirm the non-intrinsic character of the model structure given by Eq. (32). Nevertheless, a striking feature that must obviously be considered is the excellent aptitude of this model structure to fit the synthetic data yielded by the DNLR model. For these simulations, one must introduce the values of the physical parameters that are the instantaneous modulus $\mathrm{E}^{\mathrm{u}}$ and relaxed modulus $E^{r}$. The simulations have been performed for 

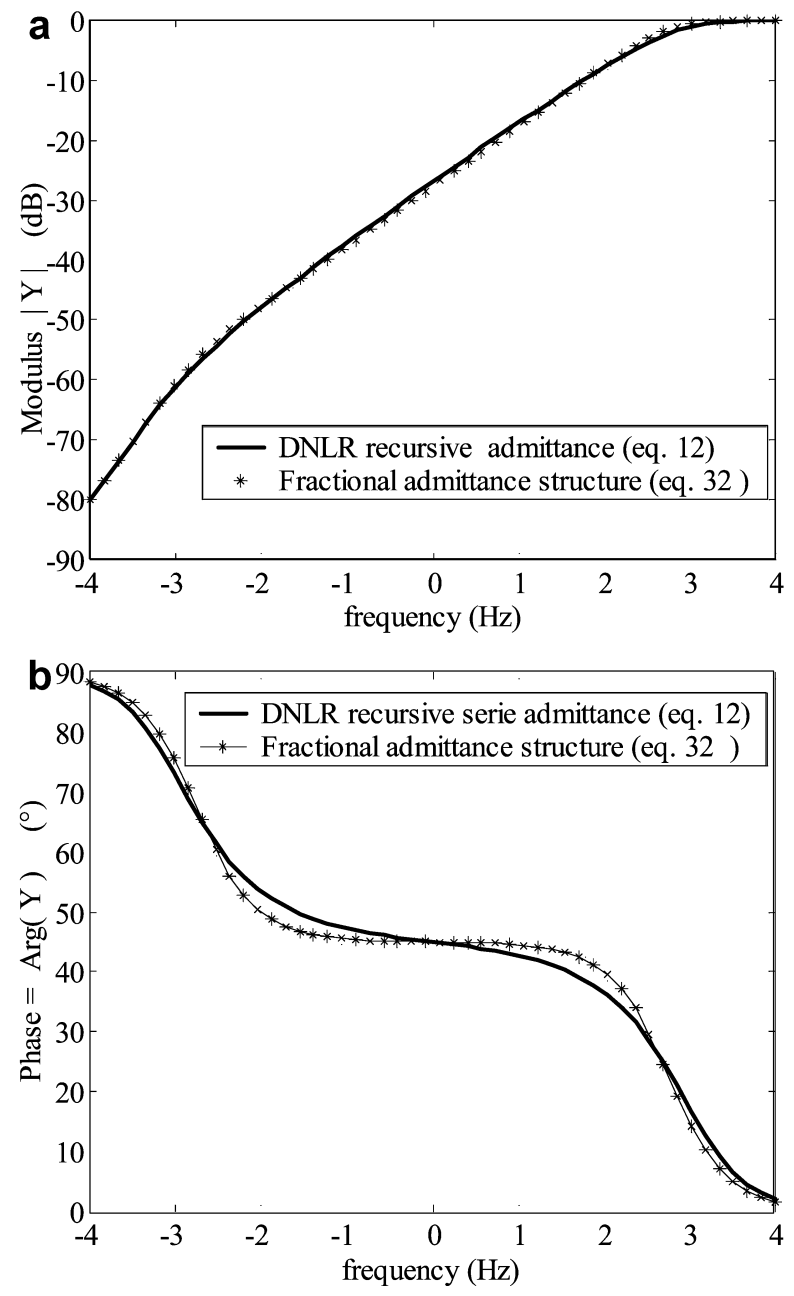

Fig. 8 a Comparison of the DNLR admittance with fractional admittance of Oustaloup: modulus vs frequency. b Comparison of the DNLR admittance with fractional admittance of Oustaloup: phase vs frequency

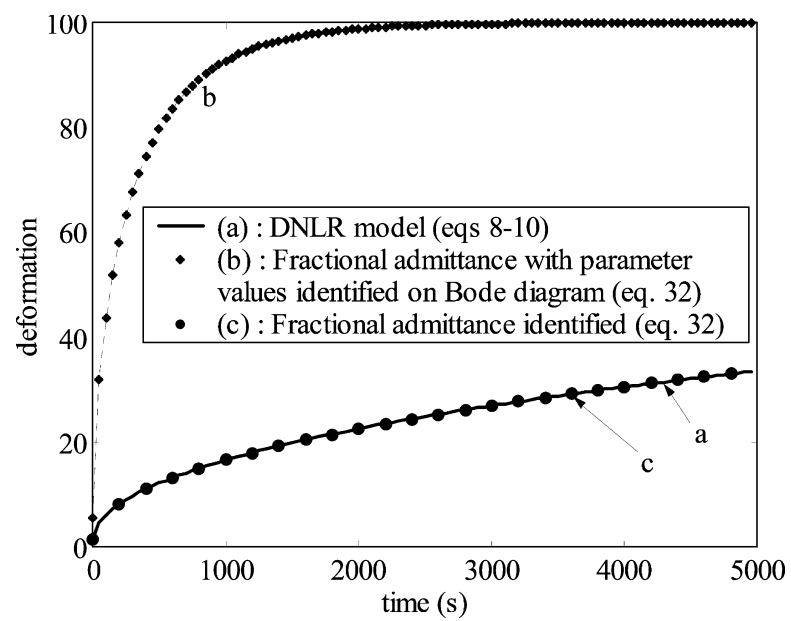

Fig. 9 Simulation of Creep experiment: comparison of DNLR model with fractional model of Oustaloup

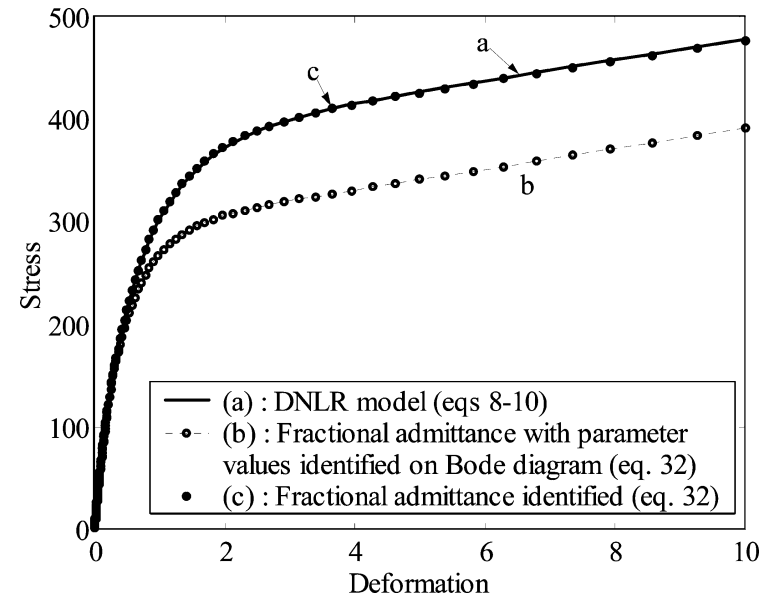

Fig. 10 Simulation of tensile load experiment: comparison of DNLR model with fractional model of Oustaloup

$E^{\mathrm{u}}=1000$ and $\mathrm{E}^{\mathrm{r}}=10$. No specific units are given here as these values do not correspond to any particular material. The values respect a ratio of 100 generally encountered for polymer relaxation in glass transition.

In Fig. 9 we show the creep experiment simulated with the DNLR model (curve a) along with the creep experiment modeled using admittance $Y_{n^{\prime}}$ where the parameter values equal those found by identification on the phase evolution curve of the Bode diagrams (curve b). Agreement is far from being exact. Then an identification procedure determines the set of parameter values that allow $\mathrm{Y}_{\mathrm{n}^{\prime}}$ to match $\mathrm{Y}^{*}$ an perfectly (curve c). Different values are then found for the parameter vector estimates: $\mathrm{n}^{\prime}=0.473, \omega_{\mathrm{b}}=1.57 \mathrm{e}^{-5}, \omega_{\mathrm{h}}=1091 \mathrm{~Hz}$.

The same applies with a constant rate tensile deformation experiment. Curve a of Fig. 10 corresponds to the DNLR simulation when considering a deformation rate of $\dot{\varepsilon}=10^{-3} \mathrm{~s}^{-1}$. Curve b corresponds to model structure $Y_{n^{\prime}}$ with the parameter vector set equal to the one estimated on Bode diagrams. Curve $\mathrm{c}$ is the result of an identification procedure of parameters $\mathrm{n}^{\prime}, \omega_{\mathrm{b}}$, and $\omega_{\mathrm{h}}$ from curve a. We found $\mathrm{n}^{\prime}=0.4, \omega_{\mathrm{b}}=1 . \mathrm{e}^{-3} \mathrm{~Hz}$, $\omega_{\mathrm{h}}=654 \mathrm{~Hz}$.

In both experiments considered above, the value of $\omega_{\mathrm{h}}$ that is identified cannot be considered as relevant because this parameter has a very small sensitivity in the model (i.e., many different estimates can be obtained depending on the initial value). The conclusions can then be:

- The structure model $Y_{n^{\prime}}$ although unable to match the recursive DNLR model on Bode diagrams, cannot be expected therefore to be intrinsic or universal but this structure can reproduce dual experiments with very good accuracy.

- One can also note that the values obtained for parameters $\omega_{\mathrm{b}}$ and $\omega_{\mathrm{h}}$ do not exactly correspond to 
the values of the bounds fixed by the DNLR model. A maximum relaxation time of $1000 \mathrm{~s}$ does not lead to $\omega_{\mathrm{b}}=0.001 \mathrm{~s}$ and the minimum relaxation time of 1. $\mathrm{e}^{-3} \mathrm{~s}$ ( six decades under $\tau_{\max }$ ) does not lead to a high frequency limit of $\omega_{\mathrm{h}}=1 \mathrm{kHz}$. This may be explained by the smoothing character of Eq. (32) that is not able to model the behavior around the extremities of the spectrum as demonstrated in Fig. 8b. This problem can be seen as equivalent to the one discussed in the paragraph above where Oldham and Zoski experienced the necessity of adding two extra RC cells at the limits of their domino ladder chain plus an extra resistance, in order to adapt their analog fractional derivative operator to the necessary limiting number of processes (electronic components).

Oustaloup too explicitly mentioned that both $\omega_{\mathrm{b}}$ and $\omega_{\mathrm{h}}$ values generally need to enlarge the relaxation spectrum considered.

Although not intrinsic, the structure model given by Eq. (32) offers a direct link with the expression of some fractional constitutive laws linking both stress and strain variables but this does not correspond to the simple generalization of integer-order derivative laws as classically expressed in the literature.

Using Eq. (32), one can write the anelastic rate of deformation (creep experiment) as

$$
\begin{aligned}
\frac{\overline{\dot{\varepsilon}}^{(a)}(s)}{\bar{\sigma}(s)} & =Y_{n^{\prime}}(s)=s^{a} \frac{s\left(1+\frac{s}{\omega_{h}}\right)^{n^{\prime}-1}}{\left(1+\frac{s}{\omega_{b}}\right)^{n^{\prime}}} \\
& =s^{a} s\left(1+\frac{s}{\omega_{h}}\right)^{n^{\prime}-1}\left(1+\frac{s}{\omega_{b}}\right)^{-n^{\prime}}
\end{aligned}
$$

and the total expression of the behavior law (including the instantaneous part) is in the Laplace domain:

$\frac{\bar{\varepsilon}(s)}{\bar{\sigma}(s)}=s^{u}+s^{a}\left(1+\frac{s}{\omega_{h}}\right)^{n^{\prime}-1}\left(1+\frac{s}{\omega_{b}}\right)^{-n^{\prime}}$

Let us introduce the definition of an implicit noninteger derivative of order $\mathrm{n}$ as

$$
\left(\frac{d}{d t}\right)_{i m p l, \tau}^{n} f(t)=\left(\frac{d}{d t}\right)^{n}[f(t) \exp (t / \tau)]
$$

It is referred to as the implicit non-integer derivative because it does not apply to $\mathrm{f}(\mathrm{t})$ but to the product of $\mathrm{f}(\mathrm{t})$ with an exponential of time constant $\tau$.

In the Laplace domain, this operator leads to a transmittance of the Davidson-Cole form

$F(s)=\frac{1}{(1+\tau s)^{n}}=\frac{1}{(1+s / \omega)^{n}}$

Then if inverse this definition and the translation property of the Laplace transform (Oustaloup 1995), it is easy to obtain a constitutive law expressed in the following form:

$$
\begin{aligned}
\varepsilon(t)= & s^{u} \sigma(t) \\
& +s^{a} \omega_{h}^{1-n} e^{-\omega_{h} t}\left(\frac{d}{d t}\right)_{i m p l, \omega_{h}}^{n-1}\left[\omega_{b}^{n} e^{-\omega_{b} t}\left(\frac{d}{d t}\right)_{i m p l, \omega_{b}}^{-n} \sigma(t)\right]
\end{aligned}
$$

This form is obviously not as simple as the generalization made from classical models by simply substituting fractional derivatives for integer ones (fractional Zener or Maxwell models). However, this type of fractional operator was recently put forward as an efficient way of modeling relaxation:

- In the work of Friedrich and al. (1999) first, where it is introduced empirically by multiplying any relaxation function obtained from a fractional model by a cut-off function $\exp \left(-\mathrm{t} / \lambda_{\mathrm{m}}\right)$. The authors show how it improves experimental fitting in guaranteeing the fast release of stresses for $\mathrm{t}>\lambda_{\mathrm{m}}$.

- In the textbook of LeMéhauté et al. (1998) (chapter 5) secondly, where the operator is called 'offset fractional diff-integration operator'. It is obtained theoretically when an exponentially damped fractal cantor set is considered as the distribution on which a regularization (averaging) convolution process (the integral formulation) is performed using the physical test function $(\sigma(\mathrm{t})$ in our case) as kernel.

As a conclusion, we should remember that this kind of behavior law (Eqs. 28 and 37) originates from the smoothing of admittance models resulting from recursive schemes expressed as a finite summation over processes bounded in time. Although non hazardously efficient in terms of fitting abilities of dynamical experiments, their non-intrinsic character (non-constant values of the parameters) has clearly been pointed out in the analysis of the Bode diagrams. Nevertheless, this constitutes a direct link justifying the use of non-integer derivatives in the elaboration of constitutive laws in rheology. This formulation is supposed to become intrinsic and exact only in the idealized and useless case of an infinite number of internal processes involving an unbounded spectrum of relaxation times.

\section{Summary, perspectives, and conclusions}

A model of relaxation phenomena applied to the rheology of viscoelastic materials has been developed within the framework of the Thermodynamics of Irreversible Processes in the linear range. The anelastic part of the impulse time response of the system, carrying irreversibility, has been shown to be expressed by a recursive 
series. This means that the response is made of the sum of the contribution of self-similar structures whose governing parameters, especially the time constant, follow a regular geometric progression from a component to its neighbor. This result is obtained by applying the idea of an equal entropy rate production as established by Prigogine when considering a normal distribution of fluctuations along their regression path. From this recursive concept, use can be made of non-integer derivative operators in the establishment of constitutive laws. It has nevertheless been shown that these operators can exist in more than one manner and, in any case, are not intrinsic or universal (the parameters of the operator need to be modified depending on the input applied to the system). This imperfect formulation may have been pointed out by researchers working in this area as recent works (Carpinteri and Cornetti 2002) state the necessity of introducing a so-called Local Fractional Derivative (LFD) operator to overcome, with two corrections, "some physically undesirable features of the classical definition" (subtended classical fractional calculus).

What is established nowadays is the link of the fractional operators with an underlying fractal nature of the geometry on which dynamical events occur. A regular and infinite recursive scheme for a dynamical system can be shown according to Moshrefi-Torbati and Hammond (1998) as the result of a convolution of the input time function over a fractal Cantor set (see above all LeMéhauté et al. 1998; Nigmatullin 1990). The demonstration given by Nigmatullin in his paper relies on the use of what he called recursion relations and therefore meets the present analysis. In practice one may admit, according to Mandelbrot (1982), that fractal patterns in materials need not be infinite to produce a certain class of hereditary mechanisms, and that the distribution set operating in convolution integrals may work as a band pass filter, possibly locally indexed to some appropriate scaling scheme. As LFD shows a behavior similar to the Hausdorff measure of a fractal set, one can imagine that it may effectively overcome the problems discussed in the paper.

Far from these acute, ambitious, and long-term perspectives, the conclusion at this stage of the connection shown between both approaches is that the thermodynamic DNLR model in its simplest linear version, is as efficient as FDM models: first it uses exactly the same number of parameters and second it allows (i) a consistent expression for the entropy rate to be derived, as it incorporates thermomechanic coupling effects, which is not the case for FDM and (ii) a natural extension in the non-linear range as already underlined in the text for various applications such as viscoplasticity, plasticity, aging, phase transitions, etc. This is obviously a major advantage of thermodynamically based models. From this first bridge between the two approaches, one now has to investigate the limits of the principle of entropy equipartition for the irreversible processes. The principle may also be adapted to match ideas and concepts connected to a fractal geometrical substrate. To quote the words of Nigmatullin, one could see how "the experimentally observed scale law dependence of relaxation phenomena can be matched to the 'transfer' of the fractal dimension of a fractal object to other physical quantities, generally by means of integration". However the main origin of the recursivity in the DNLR framework is essentially and simply a consequence of both the modal analysis, which leads to independent probabilities for spontaneous regressions of microstructural fluctuations, and the semi-continuous or continuous distribution of relaxation times along the log time scale, i.e., along an activation energy arrow. This work can therefore be directly connected to those carried out in the field of pure physics (Shlesinger and Klafter 1986).

\section{References}

Aharoune A, Marceron-Balland P, Cunat C (2001) The DNLR approach and relaxation phenomena. Part II. Application: volume recovery of PVAc. Comparison between the DNLR approach and some other modelings. Mech Time-Depend Mater 5:345-377

Allen DH (1985) A prediction of heat generation in a thermo-viscoplastic uniaxial bar. Int J Solids Struct 21(4):325-342
Ayadi Z, Marceon P, Schmitt JF, Cunat C (1999) Thermodynamic modeling of creep mechanical behaviour by non linear relaxation. Eur Phys J AP 6:229235

Bagley RL (1989) Power law and fractional calculus model of viscoelasticity. AIAA J 27(10):1412-1417

Bagley RL (1991) The thermorheologically complex material. Int $\mathbf{J}$ Eng Sci 29(7):797-806

Bagley RL, Torvik PJ (1986) On the fractional calculus model of viscoelastic behavior. J Rheol 30(1):133-155
Balankin AS (1997) Physics of fracture and mechanics of self-affine cracks. Eng Fracture Mech 57:135-203

Bendler JT, Shlesinger MF (1985) Dielectric relaxation via the Montroll-Weiss random walk of defect. In: Shlesinger MF, Weiss GH (eds) The wonderful world of stochastics, a tribute to E.W. Montroll. Elsevier Sciences, pp 33-46

Bendler JT, Shlesinger MF (1988) A first passage time problem for random walk occupancy. J Stat Phys 53:1069-1087 
Biot MA (1958) Linear thermodynamics and the mechanics of solids. Proceedings of the 3rd US National Congress of Applied Mechanics. ASME, pp 1-18

Carpinteri A, Cornetti P (2002) A fractional calculus approach to the description of stress and strain localization in fractal media, Chaos Solitons Fractals 13:8594

Chrysochoos A, Peyroux R (1998) Analyse expérimentale et modélisation numérique des couplages thermomécaniques dans les matériaux solides. Rev Gén Therm 37:582-606

Coleman BD, Gurtin ME (1967) Thermodynamics with internal state variables. $\mathbf{J}$ Chem Phys 47:597-613

Cunat C (1985) Approche statistique des propriétés thermodynamiques des états liquides et vitreux. Relaxation des liquides et transition vitreuse. Influence des associations chimiques. Thèse de Doctorat d'Etat

Cunat C (1988) Thermodynamic treatment of relaxation in frozen-in systems. "University" of the distribution law for relaxation times. Z Phys Chem Neue Folge 157:419-423, 425-429

Cunat C (1991) A thermodynamical theory of relaxation based on distribution of non linear processes. J Non-Cryst Solids 131-133, 196-199

Cunat C (2001) The DNLR approach and relaxation phenomena. Part I. Historical account and DNLR formalism. Mech Time-Depend Mater 5:39-65

De Donder T (1920) Leçons de thermodynamique et de chimie physique. Gauthiers-Villars, Paris

De Groot SR, Mazur P (1962) Non-equilibrium thermodynamics. North Holland Publishing, Amsterdam

Dillon OW (1962) An experimental study of the heat generated during torsional oscillations. J Mech Phys Solids 10:224 235

Enelund M, Olsson P (1999) Damping described by fading memory analysis and application to fractional derivative models, Int J Solids Struct 36:939-970

Faccio-Toussaint E, Ayadi Z, Pilvin P, Cunat C (2001) Modeling of the mechanical behavior of a nickel alloy by using a time-dependent thermodynamic approach to relaxation of continuous media. Mech Time-Depend Mater 5:125

Ferry JD (1980) Viscoelastic properties of polymers, 3rd edn. Wiley, New York

Friedrich C, Schiessel H, Blumen A (1999) Constitutive behavior modeling and fractional calculus. In: Siginer DA, DeKee D, Chabra RP (eds) Advances in the flow and rheology of non-Newtonian fluids. Elsevier, Amsterdam, pp 429-466
Gemant A (1936) A method of analyzing experimental results obtained from elasto-viscous bodies. Physics 7:17-31

Glöckle WG, Nonnenmacher TF (1991) Fractional integral operators and Fox functions in the theory of viscoelasticity. Macromolecules 24:6426-6434

Haddad A (1996), Thermodynamique de la relaxation appliquée à la modélisation du comportement des polymères sous chargements complexes: viscoélasticité en regime dynamique, fluage thermostimulé, courants thermostimulés et relaxation diélectrique. Thesis, INPL, Nancy

Heymans N, Bawens JC (1994) Fractal rheological models and fractional differential equations for viscoelastic behaviour. Rheol Acta, 33:210-219

Kleiser T, Bocek MX (1986) The fractal nature of slips in crystals. Z Metallkd 77:582-587

Kluitenberg GA (1962) Thermodynamic theory of elasticity and plasticity. Physica 28:217-232

Koeller RC (1984) Applications of fractional calculus to the theory of viscoelasticity. J Appl Mech 51:299-307

Kovacs AJ, Aklonis JJ, Hutchinson JM, Ramos AR (1979) Isobaric volume and enthalpy recovery of glasses. A transparent multiparameter theory. J Polym Sci Phys 17:1097

Kuiken DC (1994) Thermodynamics of irreversible processes. Applications to diffusion and rheology. Wiley, Chichester

LeMéhauté A, Nigmatullin RR, Nivanen L (1998) Flèches du temps et géométrie fractale. Hermès, 2nd edn

Lesieutre GA, Mingori DL (1990) Finite element modeling of frequency-dependent material damping using augmenting thermodynamic field. J Guidance Control Dynam 13:1040-1050

Lion A (1997) On the thermodynamics of fractional damping elements. Continuum Mech Thermodyn 9:83-96

Mainardi F (1994) Fractional relaxation in anelastic solids. J Alloys Compd 211/ 212:534-538

Mandelbrot BB (1982) The fractal geometry of nature. Freeman, NY

Maugin GA, Muschik W (1994) Thermodynamics with internal variables. Part I General concepts. J Non-Equilib Thermodyn 19:217-249

Meixner J (1949) Thermodynamik und Relaxationsserscheinungen. Z Naturforsch 4a:594-600

Moshrefi-Torbati M, Hammond JK (1998) Physical and geometrical interpretation of fractional operators. J Franklin Inst 335B(6): 1077-1086

Mosolov A (1994) Singular fractal functions and mesoscopic effects in mechanics. Chaos Solitons Fractals 4:2093-2102
Nigmatullin RR (1990) Fractional integral and its physical interpretation. $\mathbf{J}$ Theor Math Phys 90(3):242-251

Nonnenmacher TF, Glöckle WG (1991) A fractional model for mechanical stress relaxation. Philos Mag Lett 64(2):89-93

Nowick AS, Berry BS (1972) Anelastic relaxation in crystalline solids. Academic Press, NY

Nutting PG (1921) A new generalized law of deformation. J Franklin Inst 191:679-685

Oldham KB, Zoski CG (1983) Analogue instrumentation for processing polarographic data. J Electroanal Chem 157:27-51

Onsager L (1931) Reciprocal relations in irreversible processes I. Phys Rev 37:405-426; Reciprocal relations in irreversible processes II. Phys Rev 38:2265-2279

Oustaloup A (1995) La dérivation non entière. Théorie, synthèse et applications. Hermès

Padovan J, Guo Y (1988) General response of viscoelastic systems modeled by fractional operators. J Franklin Inst 325:247-275

Prigogine I (1968) Introduction à la thermodynamique des processus irréversibles. Monographies Dunod, Paris

Rahouadj R, Cunat C (2001a) A nonlinear viscoelastic model based on fluctuating modes. In: Lemaître J (ed) Handbook of materials behavior models, vol I Deformations of materials. Academic Press, pp 107-116

Rahouadj R, Cunat C (2001b) Physical aging and glass transition of polymers. In: Lemaître $\mathbf{J}$ (ed) Handbook of materials behavior models, vol III. Multiphysics behaviors. Academic Press, pp 944-954

Rogers L (1983) Operators and fractional derivatives for viscoelastic constitutive equation. J Rheol 27(4):351-372

Rouse PE (1953) The theory of the linear viscoelastic properties of dilute solutions of coiling polymers. J Chem Phys 21(7):1272-1280

Schapery RA (1966) A theory of non-linear thermoviscoelasticity based on irreversible thermodynamics. Proceedings of the 5th US National Congress of Applied Mechanics. ASME, pp 511-530

Schiessel H, Blumen A (1993) Hierarchical analogues to fractional relaxation equations. J Phys A Math Gen 26:50575069

Schiessel H, Metzler R, Blumen A, Nonnenmacher TF (1995) Generalized viscoelastic models: their fractional equations with solutions. J Phys A Math Gen 28:6567-6584

Scott-Blair G, Caffyn JE (1949) An application of the theory of quasi-properties to the treatment of anomalous strainstress relation. Philos Mag 40:80-94 
Shlesinger MF, Klafter J (1986) The nature of temporal hierarchies underlying relaxation in disordered systems. In: Pietronero L, Tosatti E (eds) Fractals in physics. Elsevier Sciences

Stastna J, De Kee D, Powley M, Schümmer P, Otten B (1990) Fractal-time stochastic processes and dynamic functions of polymeric systems. Rheol Acta 29:137144
Tauchert TR (1967) The temperature generated during torsional oscillations in polyethylene rods. Int J Eng Sci 5:353365

Tisza L (1966) Generalized thermodynamics. MIT Press, Cambridge, MA

Tshoegl NW (1989) The phenomenological theory of linear viscoelastic behavior. An introduction. Springer, Berlin Heidelberg New York
Williams ML (1964) Structural analysis of viscoelastic materials. AIAA J 2(5):785808

Zaiser M, Hähner P (1999) The flow stress of fractal dislocation arrangements. Mater Sci Eng A270:299-307 